\title{
DIETER BIRNBACHER
}

\section{Kausalität von Unterlassungen - Dilemmata und offene Fragen ${ }^{1}$}

\author{
„... diese Unterlassung hat sich als schicksalhaft erwiesen.“
}

Werner (2004: 113)

\section{Das Ausgangsdilemma}

Ein viel zitierter Satz aus Ludwig Wittgensteins Philosophischen Untersuchungen ( $\$ 123)$ lautet: „Ein philosophisches Problem hat die Form: ,Ich kenne mich nicht aus. “ Danach sind philosophische Probleme gedankliche Knoten, die es zu entwirren gilt. Ein philosophisches Problem zu lösen heißt, Klarheit zu gewinnen, wo vorher Unklarheit war wobei die gewonnene Klarheit gelegentlich auch darin bestehen kann, zu erkennen, dass ein Problem - jetzt oder auf Dauer - unlösbar ist.

Die Kausalität von Unterlassungen - und von negativen Ereignissen allgemein - ist ein solcher Knoten. Was sich in diesem Punkt zu einem schwer zu entwirrenden Knäuel verschlingt, ist ein Gemenge von divergierenden und letztlich unvereinbaren Überzeugungen. Auf der einen Seite gehen wir im Alltagsdenken - und mehr noch im Alltagssprachgebrauch - relativ problemlos davon aus, dass das Nichtausführen bestimmter Handlungen die Rolle eines Kausalfaktors übernimmt. Diese Tendenz ist besonders immer dann ausgeprägt, wenn es sich bei der nicht ausgeführten Handlung um etwas moralisch oder rechtlich Erfordertes oder um etwas Erwartetes handelt, das ausbleibt. So führen Recht und Alltagsmoral einen bestimmten Unfall bei Glatteis unter anderem oder wesentlich auf die Tatsache zurück, dass der zuständige Hauseigentümer versäumt hat, auf dem Gehweg vor seinem Haus zu streuen, und damit auf ein Unterlassen. Nicht nur das Strafrecht kennt intentionales, vorsätzliches und fahrlässiges Unterlassen, auch im moralischen Alltag machen wir demjenigen, der in bestimmten Situationen, in denen anderen Gefahr droht, nicht eingreift, obwohl er eingreifen könnte und ihm ein Eingreifen zumutbar ist, einen Vorwurf. Es scheint, dass die Berechtigung dieses Vorwurf wesentlich auch davon abhängt, dass zwischen dem Unterlassen einerseits und dem eintretenden Schaden eine Kausalrelation besteht - dass das Nichteingreifen kausal zu dem Schadenseintritt beiträgt und der Schaden durch das unterlassene Eingreifen zumindest mitbewirkt ist.

Auf der anderen Seite wird man an den geläufigen Redeweisen von Unterlassungen, die etwas „bewirken“ oder „hervorrufen“, aber leicht irre - insbesondere dann, wenn man ihnen gedanklich näher ,,auf den Leib rückt“. Wie kann - so fragt man sich dann - ein

\footnotetext{
1 Dieter Birnbacher leitet das auf den Zeitraum von drei Jahren angelegte Teilprojekt „Kausalität von Unterlassungen“ im Rahmen der DFG-Forschergruppe „Erklärungen, Kausalität, Gesetze und Dispositionen am Schnittpunkt von Wissenschaften und Metaphysik“ (Sprecher: Univ.-Prof. Dr. Andreas Hüttemann, Westfälische Wilhelms-Universität Münster).
} 
Nichthandeln für irgendetwas ursächlich sein? Wie kann ein Nichtereignis (darauf läuft ein „negatives Ereignis“ ja hinaus) ein Ereignis ,bewirken“? Postuliert man nicht, indem man negativen Handlungen und anderen negativen Ereignissen kausale Wirksamkeit unterstellt, eine Art creatio ex nihilo? Nur aus etwas, so könnte man meinen, kann etwas entstehen, nicht aus nichts. So hat denn auch der Rechtswissenschaftler Karl Larenz ganz konsequent gemeint, Unterlassungen könnten ,für nichts kausal sein“. ${ }^{2}$ Der Eindruck, sie könnten es, sei eine Illusion, die sich möglicherweise den sprachlichen Ausdrucksweisen verdanke, derer wir uns zur Beschreibung von Unterlassungen bedienen. Diese enthalten ja gewöhnlich keine Negationspartikel, die auf die negative Beschaffenheit des Gemeinten hinweisen, sondern beschreiben Unterlassungen so, als handelte es sich um Handlungen oder Ereignisse, zum Beispiel Fehlen, Schweigen, Vergessen, Vernachlässigen. ${ }^{3}$

\section{Die skeptische Lösung und ihre Probleme}

Aus dieser Perspektive ergibt sich allerdings ein gravierendes und missliches Problem: Solange Verantwortlichkeit daran geknüpft wird, dass zwischen einem Verhalten und bestimmten Verhaltensfolgen eine irgendwie geartete kausale Verknüpfung besteht, hat diese Sichtweise zur Folge, dass niemand für die Folgen von Unterlassungen verantwortlich sein kann. Wer die Auffassung vertritt, dass Unterlassungen nicht kausal sein können, muss entweder bestreiten, dass Verantwortlichkeit Kausalität voraussetzt, oder er muss behaupten, dass eine rein negative Verantwortung (durch Unterlassen) vernünftigerweise nicht zugesprochen werden kann. Beide Alternativen scheinen gleichermaßen unannehmbar. Zumindest in der Moral und im Strafrecht gehen wir im Allgemeinen davon aus, dass das Zusprechen von Verantwortlichkeit nur insoweit legitim ist, als derjenige, der verantwortlich gemacht wird, an dem Zustandekommen des Schadens - beziehungsweise im analogen Fall des Verdienstes für ein erwünschtes Ereignis - kausal (direkt oder indirekt) zumindest beteiligt war. Wir wehren uns mit gutem Grund dagegen, für Ereignisse verantwortlich gemacht zu werden, an deren Zustandekommen wir keinen Anteil hatten, ebenso wie wir es als verfehlt empfinden, für erfreuliche Dinge gelobt zu werden, zu denen wir in keiner Weise beigetragen haben. Die andere Alternative scheint aber ebenso unappetitlich, insbesondere bei drastischen Fällen des Unterlassens, in denen jemand einen anderen $a b$ sichtlich durch Nichteingreifen zu Schaden kommen lässt. So werden vor Strafgerichten immer wieder Fälle von Mord durch Unterlassen verhandelt, die zum Beispiel dadurch möglich werden, dass das Opfer von bestimmten Hilfsmitteln (wie Insulin) abhängig ist, die es sich nicht selbst beschaffen kann, und ein anderer diese ihm gezielt vorenthält.

Eine Zeit lang bediente man sich in der deutschen Strafrechtswissenschaft zur Auflösung dieses Dilemmas einer Argumentationsstrategie, die kaum mehr ist als ein verbaler Trick: Man sprach von der ,hypothetischen Kausalität“ beziehungsweise der „Quasi-Kausalität“ von Unterlassungen. Unterlassungen seien ,hypothetische Ursachen“ insofern, als ein der jeweiligen Unterlassung entsprechendes Handeln den Schaden verhindert hätte. Auch wenn es ausgeschlossen ist, der nicht ausgeführten Handlung h Kausalität zuzuschreiben, lasse sich das Verhältnis zwischen der Unterlassung und der durch sie scheinbar bewirkten Schadensfolge w (= Wirkung) dennoch mithilfe von Kausalitätsbegriffen analy-

\footnotetext{
2 Larenz (1953: 686).

3 Vgl. Birnbacher (1995: 24 ff.).
} 
sieren, nämlich so, dass die Handlung $\mathrm{H}$, wenn sie ausgeführt worden wäre, Nicht-W zur Folge gehabt hätte. Dieses letztere Bewirken wäre ein ,echtes“ Bewirken gewesen, da es sich ja in diesem Fall um eine positive Ursache handelte.

Bei Lichte besehen ist dieser Rettungsversuch allerdings untauglich und die Redeweise von ,hypothetischer Kausalität“ irreführend. Die „echte“ Kausalität der entsprechenden Handlung existiert ja lediglich in der Fantasie und nicht in der Wirklichkeit. Real findet diese Handlung gerade nicht statt. „Hypothetisch“ ist in der Zusammensetzung „hypothetische Kausalität" ein Ausdruck, der sein Bestimmungswort nicht qualifiziert, sondern teilweise aufhebt (er ist in einem spezifischen Sinn „synkategorematisch“). Eine ,hypothetische Kausalität" ist keine Variante von Kausalität, sondern eine Variante von Nichtkausalität. Hypothetische Kausalität steht zu echter Kausalität in demselben Verhältnis wie vermeintliche Wahrheit zur Wahrheit.

Das ist nicht der einzige Versuch geblieben, die Verantwortlichkeit für Unterlassungen zu ,retten“, ohne Unterlassungen ,echte“ Kausalität zuzuschreiben. Zwei weitere Versuche, das Dilemma zwischen Nichtverantwortlichkeit und Kausalität zu umgehen, setzen anders als der erste Versuch nicht bei hypothetischen Konstruktionen, sondern bei zwei positiven Faktoren an, die in der Situation, in der jemand etwas unterlässt, beteiligt sind: die erste bei den zum gleichen Zeitpunkt ausgeführten Handlungen, ${ }^{4}$ die zweite beim innerem Unterlassungsentschluss, also einem inneren Handeln. ${ }^{5}$ Beide Versuche sind allerdings ebenfalls mit erheblichen Schwierigkeiten konfrontiert.

Der erste Vorschlag läuft darauf hinaus, das Unterlassen von h mit den Handlungen zu identifizieren, die der jeweilige Akteur ausführt, während er h unterlässt. Nehmen wir an, A fährt in Urlaub, ohne jemanden zu beauftragen, seine Pflanzen zu begießen. B könnte As Pflanzen gießen, unterlässt dies jedoch. Die Pflanzen vertrocknen, bevor A aus dem Urlaub zurückkehrt. Hat Bs Verhalten kausal zum Vertrocknen von As Pflanzen beigetragen? Diesem Ansatz gemäß sehr wohl. Allerdings nicht dadurch, dass er As Pflanzen nicht begossen hat (was ihm möglich und zumutbar gewesen wäre), sondern dadurch, dass er seiner gewohnten Arbeit nachgegangen ist, ohne As Pflanze zu begießen. Wenn B für sein Unterlassen verantwortlich gemacht wird, dann nicht insofern, als er bestimmte Dinge nicht getan hat, sondern insofern, als er bestimmte Dinge getan hat, ohne bestimmte andere Dinge zu tun. Was das Vertrocknen von As Pflanzen bewirkt, ist nicht, dass B etwas nicht getan hat, sondern dass er etwas anderes getan hat.

Dieser Umgehungsversuch scheint auf den ersten Blick vielversprechend, weist aber bei näherem Hinsehen Probleme auf. Ein erstes Problem ist, dass nicht in jedem Fall eines Unterlassens ein alternatives Handeln gegeben sein muss. Zwar ist Unterlassen nicht denkbar ohne ein handlungsfähiges Subjekt. Aber dieses Subjekt braucht nicht zu handeln, während es etwas unterlässt, weder im Sinne eines äußeren noch im Sinne eines inneren Handelns. Wie Gross selbst an einer Stelle einräumt: „Some crimes of omission may be committed when all is quiet on the mental front. "6 Ein zweites Problem ist, dass dieser Vorschlag die Pointe der Verantwortungszuweisung für Unterlassungen verfehlt: Auch dann, wenn der Unterlassende handelt, während er die fragliche Handlung h unterlässt, geht es bei der Verantwortungszuweisung für die Folgen von Nicht-H nicht um diese anderen

\footnotetext{
4 Vgl. Gross (1979).

5 Vgl. Trapp (1988).

6 Gross (1979: 62).
} 
Handlungen. Um diese geht es allenfalls, wenn die Ausführung der anderen Handlungen mit der Ausführung von h unvereinbar ist. Diese Bedingung ist jedoch nicht durchgehend erfüllt. Dass B seiner gewohnten Arbeit nachgeht, braucht mit dem Begießen von As Pflanzen nicht unvereinbar zu sein. In allen diesen Fällen sind jedoch Bs Handlungen für das Vertrocknen von As Pflanzen nicht erklärungsrelevant.

Noch offenkundiger sind die Unzulänglichkeiten des zweiten Umgehungsversuchs, der das kausale Moment der Unterlassung in der Unterlassungsintention lokalisiert. Zwar kann man die ausdrückliche Absicht, h nicht auszuführen, als eine innere Handlung auffassen und damit als ein ,,positives“ Ereignis, das auch für den Kausalitätsskeptiker als ursächlich in Frage kommt. Aber die überwiegende Zahl der Handlungen, die wir nicht ausführen, obwohl wir sie ausführen könnten, ist nichtwillentlich. Zwar erfordert die Redeweise von „Unterlassen“ in der Regel, dass derjenige, der etwas unterlässt, weiß oder zumindest vermutet, dass er durch ein Eingreifen eine bestimmte Folge verhindern könnte. Es erfordert jedoch nicht, dass, wenn er diesen Eingriff unterlässt, dies in einem Sinne will, der einen eigenständigen Entschluss oder Willensakt beinhaltet. Auch wenn Unterlassungen analog zu Handlungen willentlich steuerbar sein müssen, heißt das nicht, dass sie in jedem einzelnen Fall durch einen Willensakt gesteuert sind. Wie das Handeln bedarf auch das Unterlassen keiner besonderen Willensakte. Entscheidend für die Handlungsartigkeit von Handlungen und Unterlassungen ist lediglich, dass sie im Bedarfsfall willentlich steuerbar sind, zum Beispiel dann, wenn sich während eines Verhaltensablaufs Umstände ergeben, die eine sofortige Verhaltensmodifikation erforderlich machen. Für den genannten Beispielfall: Wenn B es unterlässt, As Pflanzen zu begießen, heißt das nicht notwendig, dass B sich dazu entscheidet, As Pflanzen nicht zu begießen. Er unterlässt es auch dann, As Pflanzen zu begießen, wenn dies unwillentlich geschieht. Noch ein weiterer Grund spricht gegen diese Konstruktion: Selbst dann, wenn B sich bewusst dazu entschließt, eine Handlung h nicht auszuführen, macht dieser Entschluss allenfalls einen Teilaspekt und nicht schon das Ganze der Unterlassung aus. Damit B es unterlässt, h zu tun, reicht es nicht, dass er sich dazu entschließt, h nicht auszuführen. (Es reicht ebenfalls nicht, dass er den Versuch unternimmt, h nicht auszuführen.) Entscheidend ist, dass B tatsächlich $\mathrm{h}$ nicht ausführt.

Falls aber diese (und einige andere in der Literatur vorgeschlagene, noch artifiziellere) Hilfskonstruktionen zu wenig plausibel sind, um die Unterlassungen abgesprochene Kausalität zu ersetzen, ist die skeptische Position mit einigermaßen harschen Konsequenzen konfrontiert. Soweit Folgenverantwortung, wie in der Moral und im Strafrecht durchweg vorausgesetzt, an Kausalität gebunden ist, kann niemand für etwas, was er durch Handeln hätte verhindern können, verantwortlich sein. Folgenverantwortung wäre auf das beschränkt, was jemand - direkt oder indirekt - durch Handeln herbeiführt. Er wäre nur für das verantwortlich, was er durch ein entsprechendes Unterlassen hätte verhindern oder vermeiden können.

Die skeptische Position hat möglicherweise noch eine weitere problematische, in der Literatur, soweit ich sehe, bisher übersehene Konsequenz: Falls Unterlassungen für die Position von Ursachen nicht in Frage kommen, sollten sie auch für die Position von Wirkungen nicht in Frage kommen. Wenn Unterlassungen als Ursachen ein Nichts sind, das nichts bewirken kann, sollten sie auch als Wirkungen ein Nichts sein, das nicht bewirkt werden kann. In diesem Fall wäre aber die skeptische Position noch ein Stück weit we- 
niger plausibel. Unterlassungen werden in Alltagsdenken und Alltagssprache mit großer Selbstverständlichkeit nicht nur an die Ursachenposition, sondern auch an die Wirkungsposition gesetzt. So etwa dann, wenn in unserem Beispielfall A vor seinem Urlaub vergisst, B mit dem Begießen seiner Pflanzen für die Zeit seines Urlaubs zu beauftragen und B dies infolgedessen unterlässt, es jedoch getan hätte, wäre er von A damit beauftragt worden. In diesem Fall wird nicht nur die Ursache, sondern auch die Wirkung als ein Nichthandeln bei bestehender Handlungsmöglichkeit, also als ein Unterlassen aufgefasst.

Offenkundig hängt die Möglichkeit oder Unmöglichkeit, Unterlassungen als Wirkungen - von Unterlassungen oder anderen Ursachen - aufzufassen, davon ab, welche Gründe dafür bestimmend sind, Unterlassungen den Status von Ursachen streitig zu machen. Nur wenn diese Gründe auch auf Unterlassungen als Wirkungen anwendbar sind, besteht in dieser Hinsicht Symmetrie. In der Tat haben keineswegs alle Positionen, die Unterlassungen als ,echte“ Ursachen ausschließen, diese Symmetrie zur Konsequenz. Eine ältere Auffassung von Kausalität, die von David Hume im 18. Jahrhundert attackiert worden ist, besagt, dass kausale Wirksamkeit eine spezifische „Kausalkraft“ beinhaltet. Diese darf dabei nicht mit der „Kraft“ verwechselt werden, von der die Physik spricht. Die gemeinte „Kausalkraft“" soll zwar auch bei der Kraftübertragung im physikalischen Bereich wirksam werden, darüber hinaus aber auch in nichtphysikalischen Kausalbeziehungen wie etwa der kausalen Einwirkung des Willens auf Gedankenoperationen. Geht man von einer derartigen - empirisch, wie Hume gesehen hat, nicht aufweisbaren, aber immerhin metaphysisch postulierbaren - Kausalkraft sui generis aus, scheint es nicht abwegig, sie Unterlassungen zwar in der Rolle von Ursachen, nicht aber in der Rolle von Wirkungen abzusprechen. Wenn Unterlassungen ein rein negatives Ereignis oder Nichtereignis sind, scheint das ein hinreichender Grund, ihnen eine Kausalkraft abzusprechen. Es fehlt schlicht an einem Ausgangspunkt, von dem die - reale oder vermeintliche - Kausalkraft ausgehen sowie an einem Trägermedium, das sie transportieren könnte. Demgegenüber kämen Unterlassungen aber sehr wohl als Wirkungen der Aktivität einer solchen Kausalkraft in Frage. Anders als eine physikalische Übertragung von Kraft, Impuls oder Energie ist eine „metaphysische“ Kausalkraft - sofern es sie geben sollte - nicht daran gebunden, dass auch auf der Wirkungsseite eine irgendwie geartete Veränderung eintritt. Gäbe es eine Kausalkraft, könnte sie zwar kein negatives Ereignis zum Ausgangspunkt, aber sehr wohl ein negatives Ereignis zum Endpunkt haben. Als ein metaphysischer Vorgang lässt sich seine Operation nicht in derselben Weise wie ein empirischer Vorgang empirisch verifizieren.

Allerdings liegt es der ganz überwiegenden Mehrheit der wissenschaftlichen „Kausalitätsskeptiker" fern, die Präsenz einer solchen mysteriösen, allen Kausalbeziehungen zugrunde liegenden, aber empirisch in keiner Weise aufweisbaren „Kausalkraft“ zu postulieren. Die Kausalitätsskeptiker sind in der Regel Wissenschaftsphilosophen, die entweder in Personalunion Naturwissenschaftler sind oder zumindest einer oder mehreren der Naturwissenschaften nahestehen. So ist es nicht weiter verwunderlich, dass sie bei dem Thema Kausalität, wie sich der Wahl ihrer Beispiele ablesen lässt, in erster Linie an Beispiele aus dem im weitesten Sinne physikalischen Bereich denken, etwa an die bereits von Hume diskutierten sich stoßenden Billardbälle, bei denen wir den Anstoßenden (beziehungsweise das Anstoßen des einen) als Ursache und das Sich-in-Bewegung-Setzen des Angestoßenen als Wirkung in einem zusammenhängenden Kausalprozess auffassen. Geht man von diesem Beispiel aus, liegt es nahe, Kausalität als eine Übertragung von Kraft, Impuls oder 
Energie im physikalischen Sinne zu verstehen, oder, in einer von dem Wissenschaftstheoretiker Dowe verallgemeinerten Form: als Übertragung einer physikalischen Größe. Was kausale Prozesse von zufälligen Koinzidenzen oder von bloßen Korrelationen (dem post hoc ohne propter hoc wie bei zwei aufeinanderfolgenden Symptomen eines gemeinsamen zugrunde liegenden Prozesses) unterscheidet und ihnen ihren spezifisch kausalen Charakter verleiht, ist dieser Auffassung nach die Übertragung einer physikalischen Größe. Für viele naheliegende Fälle ist diese Bedingung in der Tat erfüllt. Führt etwa die Präsenz eines Krankheitserregers in einem Organismus zu zwei Arten von Symptomen, die regelmäßig aufeinander folgen (etwa Ausschlag und Fieber), dann besteht eine solche Übertragungsbeziehung (über mehrere Zwischenschritte) typischerweise zwischen der Präsenz des Erregers und jedem der beiden Symptome, aber nicht zwischen den Symptomen selbst. Wir deuten das Nichtbestehen einer solchen Übertragungsbeziehung dann regelmäßig als Indiz dafür, dass es sich um eine bloße Korrelation handelt, die auf einen zugrunde liegenden kausalen Zusammenhang hinweist, ihn aber nicht selbst konstituiert. Ähnlich lassen sich Prioritätsfragen wie die, ob Leibniz oder Newton die Differenzialrechung erfunden hat, unter anderem dadurch klären, dass man im Fall eines Plagiatverdachts den Weg der Energieübertragung (hier: der Informationsübertragung) nachzeichnet, der möglicherweise von der wirklichen Entdeckung des einen zur scheinbaren Entdeckung des anderen geführt hat. Lässt sich ein solcher Weg nicht nachweisen oder glaubhaft machen, gehen wir davon aus, dass es sich um eine Koinzidenz kausal unabhängiger Ereignisse handelt.

Es leuchtet ein, dass sich auf dem Hintergrund einer derartigen ,physikalistischen“ Kausalitätstheorie die Redeweise von kausal wirksamen Unterlassungen in keiner Weise rechtfertigen lässt. Rechtfertigen ließe sich diese Redeweise nur dann, wenn das Unterlassen mit den zum Zeitpunkt des Unterlassens ausgeführten Handlungen identifiziert wird, die dann als Ausgangspunkt der Übertragung von Energie oder einer anderen physikalisch definierten Größe dienen könnten. Diesen Weg haben wir aber oben bereits mit Gründen zurückgewiesen. Auf dem Hintergrund der physikalistischen Form des kausalen Skeptizismus folgt jedoch nicht nur die Unmöglichkeit, Unterlassungen als Ursachen anzunehmen, sondern auch die Unmöglichkeit von Unterlassungen als Wirkungen. Anders als bei der Übertragung einer metaphysischen Kausalkraft erfordert die Übertragung einer Kraft oder einer anderen physikalischen Größe, dass sich dadurch, dass die Größe übertragen wird, an dem Ereignis, auf das sie übertragen wird, etwas ändert. Die Übertragung der Größe muss für das Wirkungsereignis einen Unterschied machen, und zwar einen im Prinzip empirisch aufweisbaren Unterschied. Die Tatsache, dass ein Ereignis durch ein anderes, vorhergehendes bewirkt ist, muss daran erkennbar sein, dass es sich in einem physikalisch beschreibbaren Zustand befindet, in dem es sich (außer bei einer Mehrfachverursachung) nicht befinden würde, wäre es nicht durch das betreffende andere Ereignis bewirkt worden. Ein Nichtereignis wie eine Unterlassung kommt jedoch für diese Rolle nicht in Frage. Von einem Nichtereignis kann man nicht sagen, dass es dadurch, dass es die Wirkung einer Ursache ist, eine Veränderung erfährt oder in seiner Beschaffenheit von einem hypothetischen anderen Ereignis abweicht. Ein Nichtereignis kann weder Veränderungen erfahren noch in seiner Beschaffenheit von einem anderen abweichen.

Das heißt nicht, dass man die skeptische Position nicht auch völlig unabhängig von einer physikalistischen Kausalitätstheorie vertreten kann. Wie gezeigt, wurde diese Auffassung bereits lange vor dem Aufkommen physikalistischer Theorien vertreten, etwa in 
der Rechtswissenschaft. Ein bekannter zeitgenössischer Vertreter des Skeptizismus, der nicht zugleich eine physikalistische Übertragungstheorie vertritt, ist etwa der australische Philosoph David Armstrong. ${ }^{7}$ Es ist allerdings nicht auszuschließen, dass auch hierbei intuitiv ähnliche Vorstellungen eine Rolle spielen wie die, die von den modernen Physikalisten expliziert werden: die unbestimmte Vorstellung von Kausalität als einem Prozess, bei dem etwas von der Ursache auf die Wirkung übertragen wird. Dieses Etwas, wie immer im Einzelnen vorgestellt, nimmt bei der Ursache seinen Ausgang, um bei dem Ereignis, das wir als Wirkung benennen, ,,anzukommen“. Erst dadurch, dass es ,ankommt“, macht es für dieses einen Unterschied.

\section{Die verallgemeinerte skeptische Lösung}

Für Vertreter der physikalistischen Vorstellung von Kausalität ist das Unterlassen zumeist nur ein Fall unter anderen. Was für Unterlassungen - also Nichthandlungen - gilt, muss in derselben Weise für negative Ereignisse allgemein gelten, das heißt so, dass nicht nur Unterlassungen, sondern alle negativen Ereignisse nicht nur als Ursachen, sondern auch als Wirkungen nicht in Frage kommen. Diese Erweiterung ist zweifellos einerseits konsequent: Unterlassungen machen nur einen winzigen Bruchteil aller formulierbaren oder denkbaren negativen Ereignisse aus. Andererseits entfernt sich diese Position beträchtlich von den Weisen, in denen wir in Wissenschaft und Alltag denken und sprechen, etwa über alle Sachverhalte, bei denen das Nichteintreten eines gewohnheitsmäßig erwarteten Ereignisses (etwa eine Zugverspätung) zum Eintreten eines Ereignisses (Tadel des Vorgesetzten) oder der Eintritt eines Ereignisses (etwa eine Impfung) zum Nichteintreten eines anderen Ereignisses (Grippeinfektion) zu führen scheint. In allen diesen Fällen besteht entweder die Ursache oder die Wirkung oder beide aus einem negativen Ereignis, das heißt einem Nichtereignis. Wie geht der Skeptiker mit solchen Fällen um? Muss er für solche Fälle nicht radikal „quer“ zum common sense denken, indem er die entsprechenden Denk- und Redeweisen als eine Art pragmatisch motivierter Simplifizierung auffasst, als eine folk metaphysics oder eine façon de penser, der unter Gesichtspunkten der Denkökonomie eine gewisse praktische Berechtigung nicht abzusprechen ist, die jedoch - und allein das ist metaphysisch entscheidend - keinerlei fundamentum in re hat? Negative Ursachen sind nur scheinbar „echte“ Ursachen, negative Wirkungen nur scheinbar „echte“ Wirkungen.

Die Skeptiker haben diese radikale Abweichung vom common sense gelegentlich mit verbalen Wendungen verdeckt, die es so erscheinen lassen, als bestünde ihnen zufolge zwischen negativen Ereignissen doch am Ende eine bestimmte Art von Kausalität. Wie Diplomaten haben sie den Dissens gewissermaßen hinter einem Formelkompromiss versteckt. Ähnlich wie lange Zeit die Strafrechtswissenschaftler bei strafbaren Unterlassungen von „hypothetischer Kausalität“, haben sie es sich einfallen lassen, von negativen Ursachen als „Ursachen im erweiterten Sinn“8 oder als „Quasi-Ursachen“9 ${ }^{\text {" }}$ zu sprechen - während sie allerdings gleichzeitig betonen, dass es sich dabei um keine „echten“ Ursachen handelt, sondern um „Derivate“ von „,echten“ Ursachen.

\footnotetext{
7 Vgl. Armstrong (1983).

8 Fair (1979).

9 Dowe (2001).
} 
$\mathrm{Zu}$ der Redeweise von „Derivaten“ fühlen sie sich dadurch berechtigt, dass obwohl „Quasi-Ursachen“ für sich selbst nicht als Ursachen gelten können, diese sich doch in einer bestimmten Weise auf ,,echte“ Ursachen zurückführen lassen. Auch wenn eine Quasi-Ursache keine ,echte“ Ursache ist, soll sie sich dennoch jeweils nur über eine „echte“ Ursache identifizieren lassen, wobei diese eine hypothetische Rolle übernimmt. „QuasiUrsachen“ sind solche, die unter hypothetischen Bedingungen „,echte“ Ursachen wären was im Rahmen der physikalistischen Übertragungstheorie heißt, dass sie unter kontrafaktischen Bedingungen Energie oder eine andere physikalische Größe auf ihre Wirkung übertragen würden. Genau dadurch sollen sie sich von bloßen Korrelationen unterscheiden. Danach ist eine Unterlassung wie das Nichtbegießen der Pflanzen die Quasi-Ursache für das Nichtüberleben der Pflanzen genau deshalb, weil ein Begießen für das Überleben der Pflanzen kausal gewesen wäre. Nur weil zwischen Begießen und Überleben die Beziehung einer „,echten“, das heißt durch Energieübertragung bestimmten physikalischen Verknüpfung besteht, soll zwischen dem Nichtbegießen der Pflanzen und ihrem Nichtüberleben die Beziehung einer „Quasi-Kausalität“ bestehen beziehungsweise das NichtbegieBen die „Ursache im erweiterten Sinn“ für das Vertrocknen der Pflanzen sein. Analoges gilt für nichthandlungsartige negative Ursachen wie das Ausbleiben des Monsuns. Das Ausbleiben ist deshalb und nur deshalb die Quasi-Ursache der Dürre, weil sein (erwartetes) Eintreten kraft physikalischer Einwirkung die Saat zur Ausreifung bringen würde.

In ähnlicher Weise rekonstruieren die physikalistischen Skeptiker die beiden anderen möglichen Fallkonstellationen - einerseits die Konstellation, dass ein Ereignis beziehungsweise eine Handlung ein Nichtereignis beziehungsweise eine Unterlassung quasi-verursacht, andererseits die Konstellation, dass ein Nichtereignis beziehungsweise eine Unterlassung ein Ereignis beziehungsweise eine Handlung quasi-verursacht. Jedes Mal wird die Relation der Quasi-Kausalität als eine hypothetische „echte“ Kausalitätsrelation rekonstruiert, die die Bedingung einer Energieübertragung erfüllt. Dass eine Handlung ein mögliches Ereignis verhindert (Konstellation 1), kann dann in Anwendung eines Vorschlags von Dowe so rekonstruiert werden, dass die Handlung eine „echte“ Wirkung auf einen Prozess $\mathrm{x}$ ausübt, der, falls die Handlung nicht ausgeführt würde, das Ereignis durch eine „,echte“ Verursachung bewirken würde. Ein Beispiel: Der Vorgang

„Indem der Vater das Kind festhält, verhindert er, dass das Kind gegen ein fahrendes Auto rennt."

wird rekonstruiert als

„Indem der Vater das Kind festhält, wirkt er auf einen Prozess x ein (,,X“ steht dabei für „Das Kind läuft auf die Straße“). Würde er das nicht tun, würde das Kind gegen ein fahrendes Auto rennen.“

Ersichtlich beinhaltet der Vorgang des aktiven Verhinderns einer möglichen Handlung damit ausschließlich ,echte“, das heißt eine Energieübertragung beinhaltende Kausalität. Allerdings besteht die Kausalitätsrelation in diesem Fall nicht zwischen dem realen Handeln und dem realen Nichtereignis, sondern zwischen einem hypothetischen Nichthandeln und einem hypothetischen Ereignis.

Entsprechend lässt sich die Quasi-Verursachung in der zweiten Konstellation rekonstruieren. Dass eine Unterlassung ein Ereignis quasi-verursacht, lässt sich so verstehen, dass ein Prozess x stattfindet, der das Ereignis verursacht, und zugleich gilt, dass, wenn die un- 
terlassene Handlung ausgeführt würde, diese auf $\mathrm{x}$ so einwirken würde, dass das Ereignis verhindert würde. An demselben Beispiel veranschaulicht: Die Aussage

„Der Vater versäumt es, das Kind festzuhalten, und bewirkt dadurch, dass das Kind gegen ein fahrendes Auto rennt."

wird rekonstruiert als

„Es findet ein Prozess x statt (,X“ steht dabei für „Das Kind läuft auf die Straße“), der das Ereignis bewirkt. Würde die unterlassene Handlung ausgeführt, würde diese so auf $\mathrm{x}$ einwirken, dass das Ereignis verhindert wird.“

Wiederum kommt, ersetzt man das „Verhindern des Ergebnisses“ durch die entsprechende Analyse für die erste Konstellation, in der Rekonstruktion nur noch ,echte“ Kausalität vor. Sowohl das Bewirken des Unfalls durch den Prozess als auch die Einwirkung des Festhaltens auf den Prozess beinhalten die Übertragung einer physikalischen Größe. Allerdings besteht die „echte“ Kausalität auch hier wieder nur hypothetisch. Sie ist ein Gedankending und nichts, was in der Wirklichkeit vorkäme.

\section{Die Gegenposition: Ursachen als Bedingungen}

Die Frage stellt sich: Was ist eine „hypothetische“ beziehungsweise „erweiterte“ Kausalität wert? Ich meine: nicht viel - jedenfalls nicht genug, um einige der wesentlichen Funktionen der Zuschreibung von Kausalität, etwa die Zuschreibung von Verantwortung, zu tragen. „Hypothetische“ oder „Quasi-Kausalität“ sind als Derivate von Kausalität Ersatzkonstruktionen und keine „echte“ Kausalität. Zur Zuschreibung von Verantwortung ist jedoch nach überwiegender juristischer und ethischer Auffassung „echte“ Kausalität erfordert. Darüber hinaus hat die verallgemeinerte Form des kausalitätstheoretischen Skeptizismus einige kuriose und wenig akzeptable Konsequenzen. Eine davon ist die, dass eine Handlung, durch die ein anderer getötet wird, nicht mehr als kausal für den Tod des anderen aufgefasst werden kann, sondern lediglich als quasi-kausal. Der Tod ist nur scheinbar ein positives Ereignis. Genau besehen ist er ein negatives Ereignis, die Abwesenheit von Lebensprozessen.

Zusätzlich ist die physikalistische Interpretation der Kausalbeziehung selbst schwerwiegenden Einwänden ausgesetzt. Wie kann diese Konzeption Formen kausaler Einwirkung erklären, an denen nichtphysische Ereignisse beteiligt sind, etwa die Kausalität von Willensregungen auf neuronale Prozesse oder die Kausalität von neuronalen Prozesse auf das Bewusstsein? Da man Bewusstseinsereignissen keine physikalischen Maßgrößen wie Energie im physikalischen Sinne zuschreiben kann, kann keine Theorie, die Kausalität mit dem Übertrag einer physikalischen Größe verknüpft, diesen grundlegenden Arten von Kausalität gerecht werden. Auch die Leugnung des in der Redeweise von psychophysischer Kausalität vorausgesetzten Geist-Hirn-Dualismus ist dabei kein Ausweg. Denn auch wenn man (wie der Materialismus) bestreitet, dass das Bewusstsein eine eigenständige Realität neben den neuronalen Vorgängen ausmacht, so wird doch auch der Materialist nicht bestreiten wollen, dass die Hypothese eines solchen Dualismus, auch wenn sie nicht zutreffen sollte, ebenso sinnvoll und verständlich ist wie die Hypothese, dass das eine oder das andere oder beide auf das jeweils andere kausal einwirken. Solange man an der Verknüpfung von Kausalität und Übertragung einer physikalischen Größe festhält, wird 
man das Bestehen dieser Möglichkeit kaum verständlich machen können. Auch der von dem Philosophen Héctor-Neri Castañeda vorgeschlagene Ausweg, im Fall der psychophysischen Kausalität der übertragenen Maßgröße den Wert null beizulegen, ${ }^{10}$ führt nicht weiter. Die Übertragung einer Größe vom Betrag null ist ebenso wenig die Übertragung von irgendetwas wie ein Körper von der Ausdehnung null ein Körper ist.

Es scheint, dass sich diese Schwierigkeiten nur dann überwinden lassen, wenn man einen radikal anderen Ausgangspunkt wählt: wenn man sich von dem Bild verabschiedet, das sowohl Theorien einer von der Ursache ausgehenden „Kausalkraft“ als auch physikalistische Theorien entwerfen. „Echte“ Kausalität darf nicht an die Bedingung geknüpft werden, dass ein irgend gearteter „Faden“ - ein sich durchhaltendes, persistierendes Etwas - die Glieder der Ursachenkette verbindet. Das Gegenbild geht von einer anderen Leitvorstellung aus. Es entdynamisiert Ursachen und fasst sie nicht als Produktionsmittel bestimmter Wirkungen, sondern als Entstehungsbedingungen dieser Wirkungen auf. Diese Auffassung geht auf John Stuart Mill zurück, der sie nicht zuletzt aufgrund der Notwendigkeit entwarf, der tatsächlichen Praxis der kausalen Deutung von Korrelationen in den Wissenschaften gerecht zu werden. Nach dieser Auffassung wird Kausalität als ein $\mathrm{Be}$ dingungsverhältnis verstanden, durch das bestimmte Ereignisse in einem Abhängigkeitsverhältnis von bestimmten zeitlich vorangehenden Bedingungen stehen, ohne durch sie in einem allzu wörtlichen Sinne ,hervorgebracht“ werden zu müssen. Die anthropomorphen Beiklänge eines „Hervorbringens“ der Wirkung durch ihre Ursache oder Ursachen treten in den Hintergrund. Statt produziert zu werden, ergeben sie sich aus einer bestimmten Konstellation von Faktoren, wobei es dann nicht mehr entscheidend ist, ob diese positiv aus Ereignissen oder negativ aus Nichtereignissen bestehen. Fasst man das Folgen der Wirkungen auf die Ursachen als ein Sich-Ergeben statt als ein Hervorgebrachtwerden auf, fallen auch die Schwierigkeiten weg, eine Dürreperiode als Wirkung des Nichteintretens des Monsuns oder das Vertrocknen als Wirkung des Nichtbegießens aufzufassen.

Dieses alternative Bild von Kausalität hat, abgesehen davon, dass es Raum für die negative und insbesondere Unterlassungskausalität und für die entsprechende Verantwortungszuschreibung macht, den Vorzug, allen wesentlichen Funktionen der Zuschreibung von Kausalität in derselben Weise zu genügen wie die „Produktions“-Auffassung. Erstens genügt sie ebenso wie diese dem Desiderat, die Kausalität als den metaphysischen „Kitt“ aufzufassen, der die Welt zusammenhält, als den cement of the universe. ${ }^{11}$ Die Nahtstellen des kausalen Netzwerks der Welt bleiben bindungsfähig. Allerdings ist der Klebstoff, der die Fäden zusammenhält, nicht mehr durchgängig ein Zusammenhang des aktiven Bewirkens, sondern die zwischen den Phänomenen bestehenden naturgesetzlichen Verknüpfungen. Diese bestehen unabhängig davon, ob zwischen den verknüpften Phänomenen eine physikalische Größe übertragen wird (etwa im psychologischen Bereich) oder ob an der Verknüpfung ausschließlich positive Ereignisse beteiligt sind.

Zweitens erfüllt eine Konzeption von Ursachen, die diese als positive oder negative Bedingungen des Eintritts ihrer Wirkungen auffasst, alle Bedingungen, die an die epistemischen Funktionen von Ursachen zu stellen sind; das heißt, sie genügen der Bedingung, dass Kausalbeziehungen ex post kausale Erklärungen und ex ante Prognosen für die jeweiligen

\footnotetext{
${ }^{10}$ Vgl. Castañeda (1980: 99).

11 Vgl. Hume (1980: 60) sowie Mackie (1974).
} 
Wirkungsereignisse liefern. ${ }^{12}$ Der Erklärungs- und Prognoseanspruch einer Kausalaussage wird dadurch, dass eine Bedingungsstruktur an die Stelle der Hervorbringungsstruktur tritt, nicht beeinträchtigt. Unter erkenntnistheoretischen Vorzeichen bleiben Kausalaussagen legitime Antwortmöglichkeiten auf Fragen nach den zu erwartenden Folgen ex ante wie auf Warum-Fragen ex post. Dadurch, dass die Kausalaussage auch negative Wirkungen und negative Ursachen einzubeziehen erlaubt, kommt sie besonders in dieser Hinsicht geläufigen Denk- und Sprechweisen entgegen. In den meisten Kontexten befriedigt uns eine Erklärung, die für eine positive Wirkung zutreffende negative Ursachen anführt, nicht weniger als eine Erklärung, die positive Ursachen anführt, und andersherum. Für das Vertrocknen der nicht begossenen Pflanzen wird das Nichtbegießen im Allgemeinen als eine ebenso gute und zutreffende Erklärung akzeptiert wie das Nichtangefahrenwerden des auf die Straße laufenden Kindes durch das rechtzeitige Festhalten des Kindes durch den Vater. Analoges gilt für auf Kausalbeziehungen gestützte Voraussagen.

Drittens lässt sich die Auffassung des Verursachungs- als ein Bedingungsverhältnis auch mit der so genannten ,interventionistischen“ Kausalitätsauffassung in Übereinstimmung bringen. Nach der interventionistischen Kausalitätsauffassung, wie sie Gasking und von Wright vorgeschlagen haben, ${ }^{13}$ sind Ursachen als Bedingungen zu verstehen, die durch menschliches Handeln realisiert werden können oder könnten, um eine bestimmte Wirkung hervorzurufen. Das Paradigma von Kausalität ist dieser Auffassung nach das Eingreifen in Zusammenhänge mit der Absicht, bestimmte erwünschte Wirkungen zu zeitigen. Die Zuweisung von Kausalität auf nicht durch menschliches Eingreifen bedingte Prozesse wird dann als Übertragung oder Projektion dieses Urbilds auf anderweitige Prozesse gedeutet. Dass sich die Auffassung von Kausalität als Bedingungszusammenhang, der auch negative Bedingungen einschließt, mit diesem Modell vereinbaren lässt, erscheint zunächst paradox: Negative Bedingungen stellen ja typischerweise keine Interventionen, sondern allenfalls Nichtinterventionen dar. Aber es lässt sich leicht sehen, dass dieses Modell durchaus auch auf negative Ursachen (und Wirkungen) anwendbar ist. Man muss dafür „Intervention“ lediglich durch „Realisierung“ ersetzen. Die Tatsache, dass eine negative Ursache wie das Nichtbegießen einer Pflanze ihr Vertrocknen zur Wirkung hat, kann man dann so rekonstruieren, dass sie der Tatsache äquivalent ist, dass ein bewusstes Realisieren der kausalen Bedingungen (das Nichtbegießen) die Wirkung hervorruft oder hervorrufen würde. Eine Pflanze nicht zu begießen ist zwar keine Handlung, aber sie ist doch handlungsartig. Sie kann Teil von Absichten, Planungen und Strategien werden. ${ }^{14}$ Wollte man etwa das Vertrocknen der Pflanzen herbeiführen, wäre das Nichtbegießen eine zweifellos ebenso geeignete Strategie wie das Begießen der Pflanzen zu deren Nichtvertrocknen. Denkt man sich die Interventionstheorie auf mögliche Interventionen erweitert, würden auch nichthandlungsartige Ereignisse als negative Ursachen legitimiert. Obwohl der Monsun ein nichthandlungsartiges Ereignis darstellt, kann man sich sein Ausbleiben als Ursache im Sinne der Interventionstheorie denken, indem man sich vorstellt, ein Gott habe die Absicht, die Menschen durch eine Dürre zu bestrafen und bediene sich dazu des Nichteintretens des Monsuns. Alles, was dazu erforderlich ist, ist die göttliche Fähigkeit,

\footnotetext{
${ }^{12}$ Vgl. Schaffer (2004: 199).

${ }^{13}$ Vgl. Gasking (1955) sowie von Wright (1971).

${ }^{14}$ Man denke etwa an Rezepte, die die Anweisung beinhalten, bestimmte - übliche - Handgriffe nicht auszuführen oder bestimmte - übliche - Zutaten wegzulassen.
} 
den Monsun eintreten zu lassen. Ein Geist, der es regnen lassen kann, muss es auch nicht regnen lassen können.

Auch an den logischen Strukturen der Kausalrelation ändert sich nichts, fasst man Unterlassungen und andere negative Ereignisse als Bedingungen statt als Produzenten ihrer Wirkereignisse auf. Wie für positive gilt auch für negative Ursachen, dass sie nur in Ausnahmefällen für ihre Wirkereignisse kausal notwendig oder kausal hinreichend sind. Auch im Fall der besagten Pflanzen ist das Nichtbegießen weder eine notwendige noch eine hinreichende Bedingung ihres Vertrocknens. Die Pflanzen könnten auch infolge einer Krankheit vertrocknen, und sie könnten trotz Nichtbegießens dank außergewöhnlicher Umstände, etwa einer besonders feuchten Witterung, am Leben bleiben. Oder mehrere negative Kausalfaktoren könnten zusammentreffen, entweder so, dass beide für sich hinreichend, aber nicht notwendig sind (Nichtbegießen und Nichtdüngen), oder so, dass beide notwendig sind, aber keine für sich hinreichend ist (Nachsehen, wie es den Pflanzen geht, und Wasserholen). Die adäquateste bisher vorgeschlagene logische Rekonstruktion der Kausalrelation, John Mackies Konzeption der so genannten INUS-Bedingungen, nach der eine Ursache ein uneliminierbarer Teil einer kausal hinreichenden, aber nicht notwendigen Bedingung der Wirkung ist, ist gegenüber der Frage, ob es sich um eine positive oder eine negative Bedingung handelt, indifferent. Auch eine negative Bedingung kann den Part einer Ursache beziehungsweise eines ursächlichen Faktors übernehmen.

Diese Tatsache hat eine überraschend aktuelle Bedeutung, nämlich im Zusammenhang mit der so genannten ,passiven“ Sterbehilfe. Als „,passiv“ gilt diese Form der Sterbehilfe, weil sie typischer-, aber nicht notwendigerweise durch ein Unterlassen vollzogen wird. Ein Patient wird sterben gelassen, indem man darauf verzichtet, sein Leben zu verlängern, das man durch medizinische Interventionen verlängern könnte, etwa indem man eine lebensverlängernde Behandlung abbricht oder eine aufgenommene Behandlung weiterführt, aber mit einer für das Überleben nicht mehr ausreichenden Intensität. Interessant ist jedoch, dass sich viele Ärzte dagegen wehren, dass ein Behandlungsabbruch (ob vom Patienten verlangt oder aus Gründen des Patientenwohls vorgenommen) als ,passive“ Sterbehilfe und damit als Form von Sterbehilfe gelten soll. „Sterbehilfe“ legt nahe, dass der Arzt in irgendeiner Weise kausal am vorzeitigen Tod des Patienten beteiligt ist. Im Falle eines Behandlungsabbruchs oder des „Einfrierens“ einer Behandlung auf einem nicht mehr ausreichenden Niveau sterbe der Patient jedoch an seiner Krankheit und nicht an einer ärztlichen Intervention.

Der Unmut dieser Ärzte ist verständlich, aber der Sache nach unberechtigt. Denn zumindest nach der INUS-Analyse muss auch die unterlassene Weiterbehandlung als eine der Ursachen des vorzeitigen Todes des Patienten gelten. Auch wenn sie durch ein bloßes Unterlassen realisiert ist, erfüllt sie alle an eine echte Ursache zu stellenden Bedingungen. Die unterlassene Weiterbehandlung ist ein nicht eliminierbarer Teil einer hinreichenden Bedingung für den vorzeitigen Tod des Patienten. Ohne das Unterlassen wären die übrigen Bedingungen für sich nicht hinreichend.

Selbstverständlich ist sie nur eine von vielen Teilbedingungen. Aber als eine negative Teilbedingung ist sie ein ebenso vollwertiger Kausalfaktor wie die positiven Faktoren. In ihrer kausalen Rolle unterscheidet sie sich nicht von einem hypothetischen aktiven Eingreifen. 


\section{Ein neues Dilemma: Inflationierung von Ursachen}

Kann die Bedingungskonzeption der Kausalität als „Lösung“ des Dilemmas der Unterlassenskausalität gelten? Einiges spricht dafür, dass es dazu noch zu früh ist. Denn die damit verknüpfte Zulassung von Unterlassungen als Ursachen - und erst recht, in der erweiterten Form, die Zulassung von nichthandlungsartigen negativen Ereignissen als Ursachen generiert ein neues Dilemma, die Inflationierung von Ursachen.

Dieses neue Dilemma ergibt sich daraus, dass jede Handlung mit einer großen Zahl von Unterlassungen einhergeht, das heißt von Handlungen, die nicht ausgeführt werden, aber ausgeführt werden könnten. Dadurch kommt es zu einer beträchtlichen Ausweitung der negativen kausalen Verantwortlichkeit. Nicht mehr nur für die Folgen von h wäre der Akteur kausal verantwortlich, sondern auch für die Folgen der Nichtausführung von j, k, 1 usw., die er statt $h$ ausführen könnte. Sein Verhalten wäre nicht mehr nur der Ausgangspunkt der Kausalkette, die von der Handlung h ausgeht, sondern darüber hinaus einer Fülle weiterer Kausalketten, die von Nicht-j, Nicht-k und Nicht-1 ausgehen. Auf diese Weise kommt es zu einer wundersamen Vermehrung von Kausalrelationen. Während wir im Alltag eine kausale Verantwortlichkeit für das, was wir nicht tun, jemandem im Allgemeinen nur dann zuschreiben, wenn es sich bei den Folgen dessen, was wir nicht tun, indem wir h tun, um hinreichend schwerwiegende oder aus anderen Gründen bemerkenswerte Ereignisse handelt, scheint uns die Bedingungskonzeption darauf zu verpflichten, alle und noch die marginalsten Folgewirkungen dessen, was wir nicht tun, indem wir etwas Bestimmtes tun (aber tun könnten), als Wirkungen unseres Verhaltens, das heißt als von diesem Verhalten kausal bedingt aufzufassen.

Es gibt mehrere Strategien, mit diesem neuen Dilemma fertig zu werden. Eine mögliche Strategie ist die Leugnung. Sie besteht darin, abzustreiten, dass es durch die Einbeziehung negativer Ursachen zu einer Inflationierung kommt, und zu behaupten, dass, was die Anzahl der Wirkungen betrifft, positive und negative Ursache gleichauf sind. Auch positive Ursachen hätten unabsehbar zahlreiche Wirkungen, wenn man etwa an die kausalen Folgen des Urknalls denke. ${ }^{15}$ Damit wird aber die besondere Problematik der negativen Ursachen verfehlt. Diese liegt ja darin, dass - abhängig von dem jeweils zugrunde gelegten Begriff der Möglichkeit - die Zahl der infolge des Eintritts eines Ereignisses nicht eintretenden Ereignisse um Größenordnungen größer ist als die Zahl (wie immer diese bestimmt wird) der eintretenden Ereignisse.

Eine zweite Strategie ist die Beschränkung der Ursächlichkeit von Unterlassungen auf diejenigen Unterlassungen, die in der Nichtausführung der von einem Akteur erwarteten oder geforderten Handlungen bestehen. ${ }^{16}$ Danach qualifiziert sich nicht jede beliebige Nichtausführung einer Handlung, die eine Wirkung w verhindern würde, als Ursache der eingetretenen Wirkung w. Als „Ursache“ von w käme vielmehr eine Unterlassung nur soweit in Frage, als ein entsprechendes Handeln zu erwarten oder dieses aus normativen Gründen - etwa aufgrund bestimmter moralischer oder rechtlicher Normen - verlangt war. Eine solche Beschränkung der Ursächlichkeit von Unterlassungen kommt zweifellos verbreiteten Denk- und Sprechweisen entgegen. In vielen praktischen Kontexten orientieren wir uns bei der Zuschreibung von kausaler Verantwortung nicht nur an den tatsächlichen

\footnotetext{
15 So etwa Dowe (2001: 222).

${ }^{16}$ So Boniolo und De Anna (2006).
} 
Gegebenheiten, sondern auch an eigenen oder fremden Erwartungen oder Forderungen. Das gilt insbesondere für Nichthandeln als kausaler Bedingung für Abläufe, die bei einem aktiven Eingreifen hätten vermieden oder verhindert werden können. Wir meinen (und sagen) im Allgemeinen nur dann, dass jemand einen anderen sterben, in sein Unglück rennen oder im falschen Glauben gelassen hat, wenn entweder wir selbst oder andere von dem jeweiligen Akteur ein alternatives Verhalten (nämlich ein Eingreifen) erwarten oder fordern. Dies lässt sich an dem erwähnten Beispielfall verdeutlichen: Nehmen wir an, A beauftragt, bevor er in Urlaub fährt, B damit, seine Pflanzen zu begießen. B könnte As Pflanzen begießen, unterlässt dies jedoch. Aber auch die Kollegen C, D und E, die As Pflanzen begießen könnten, tun dies, da sie sich nicht für zuständig halten, nicht. Zweifellos würde die moralische Verantwortlichkeit in diesem Fall ausschließlich oder primär auf B fallen. Viele würden aber einen Schritt weiter gehen und behaupten, dass in diesem Fall auch die kausale Verantwortlichkeit ausschließlich auf B fällt. Bs Untätigkeit ist für das Vertrocknen von As Pflanzen kausal verantwortlich, weil und insofern er dafür normativ verantwortlich war beziehungsweise weil ein Tätigwerden von ihm erwartet wurde. Die durch die Annahme des Auftrags begründete Zuständigkeit beziehungsweise die vorherrschenden Erwartungen entscheiden in diesem Fall nicht nur über die moralische, sondern auch über die kausale Zurechnung. Es ist klar, dass, folgte man diesem Vorschlag, die Zahl der Unterlassungsfolgen drastisch reduziert wäre.

Die dritte Strategie ist eine verschärfte Form der zweiten. Sie besteht darin, die Merkmale des Erwartetseins oder Gefordertseins nicht nur als Kriterien anzuerkennen, die über die Kausalität von Unterlassungen entscheiden, sondern diese Merkmale bereits als Begriffsmerkmale von Unterlassungen aufzufassen. Danach läge in den fraglichen Fällen von vornherein kein Unterlassen vor. ${ }^{17}$ Man könnte nur von B, der den entsprechenden Auftrag von A übernommen hat, sagen, dass er das zugesagte Begießen von As Pflanzen unterlässt, nicht aber von C, D und E - es sei denn, der Sprecher wolle der Überzeugung Ausdruck geben, dass er das fragliche Handeln auch von C, D und E erwartet oder fordert. Danach löst sich das Inflationierungsproblem ebenfalls auf - nicht, wie bei der zweiten Strategie, durch eine quantitative Eingrenzung der Kausalitätsbeziehungen, in denen Unterlassungen stehen können, sondern durch eine quantitative Reduktion der Unterlassungen selbst.

Die Diskussion um die Tragfähigkeit dieser letzteren Strategien und das Ausmaß, in dem ihnen eine Auflösung des Dilemmas der Inflationierung von negativen Ursachen gelingt, ist noch nicht abgeschlossen. Einige der Gründe, die für diese Strategien ins Feld geführt werden, sind allzu eindeutig an pragmatischen Gesichtspunkten orientiert, um unter systematischen Aspekten zu befriedigen, etwa der Hinweis auf die sich aus der Anerkennung einer unbeschränkten Unterlassungskausalität ergebende Möglichkeit, auch der Unterlassung verbotener Mittel (etwa der Androhung von Folter) kausale Verantwortlichkeit für unerwünschte Ereignisse (Nichtgestehen) zuzuschreiben. ${ }^{18}$ Unbefriedigend an der dritten Strategie ist insbesondere, dass sie es ausschließt, von einem Unterlassen auch dann zu sprechen, wenn ein oder mehrere Akteure, die eingreifen könnten, erwägen, in einen laufenden Prozess einzugreifen, dies dann aber nicht tun, so dass sich die befürchtete Wirkung einstellt. So könnte in unserem Beispielfall C überlegen, ob er B an As Auftrag

\footnotetext{
${ }^{17}$ Vgl. Berger (2004: 344).

18 Vgl. Mack (1988: 64).
} 
erinnert oder ob er statt B As Pflanzen begießt. Nach der zweiten wie nach der dritten Strategie wäre diese Überlegung kausal irrelevant für das Eintreten der Wirkung. Nach der zweiten Strategie käme dem Nichtverfolgen dieser Erwägung keine kausale Wirksamkeit für das Ergebnis zu. Nach der dritten könnte es noch nicht einmal als ein Unterlassen aufgefasst werden.

Fazit: Auch wenn sich das erste Dilemma der Unterlassungskausalität mithilfe der Konzeption von Kausalität als Bedingungsstruktur erfolgreich und intuitiv befriedigend auflösen lässt, scheint diese Lösung nur um den Preis eines Folgedilemmas zu haben zu sein, des Dilemmas der Inflationierung von Ursachen. Anders als für das Ausgangsdilemma ist eine Auflösung des Folgedilemmas allerdings vorerst nicht in Sicht.

\section{Literatur}

Armstrong, David M. (1983). What is a law of nature? Cambridge.

BERGER, Armin (2004). Unterlassungen. Eine philosophische Untersuchung. Paderborn.

BIRnBACHER, Dieter (1995). Tun und Unterlassen. Stuttgart.

Boniolo, Giovanni und Gabriel DE ANNA (2006). „The four faces of omission“, Philosophical Explorations 9, 277-293.

CAstañeda, Héctor-Neri (1980). „Causes, energy and constant conjunctions“, in: Peter VAN INWAGEN (Hrsg.). Time and cause. Essays presented to Richard Taylor. Dordrecht, 81-108.

Dowe, Phil (2001). ,A counterfactual theory of prevention and ,causation“ by omission“, Australasian Journal of Philosophy 79, 216-226.

FAIR, David (1979). „Causation and the flow of energy“, Erkenntnis 14, 219-250.

GASKING, Douglas (1955). „Causation and recipes“, Mind 64, 479-487.

Gross, Hyman (1979). A theory of criminal justice. New York 1979.

Hume, David (1980). Abriß eines neuen Buches, betitelt: Ein Traktat über die menschliche Natur, etc./Brief eines Edelmannes an seinen Freund in Edinburgh. Hamburg.

LARENZ, Karl (1953). „Ursächlichkeit der Unterlassung“, Neue Juristische Wochenschrift 6, 686687.

MACK, Eric (1988). „Moral rights and causal casuistry“, in: Baruch A. BRODY (Hrsg.). Moral theory and moral judgments in medical ethics. Dordrecht 1988, 57-74.

MACKIE, John L. (1974). The cement of the universe. Oxford.

SCHAFFER, Jonathan (2004). „Causes need not be physically connected to their effects. The case for negative causation“, in: Christopher HITCHCOCK (Hrsg.). Contemporary debates in philosophy of science. London, 197-216.

TRAPP, Rainer W. (1988). Nicht-klassischer Utilitarismus. Eine Theorie der Gerechtigkeit. Frankfurt am Main.

WERnER, Marcus. Am Hang. Frankfurt am Main 2004.

WRIGHT, Georg Henrik VON (1971). Explaining and understanding. Ithaca, NY. 
